\title{
Cluster analysis of age-related trends of the expression of metabolically relevant genes in humans PBMCs
}

\author{
Aleksey Alekseev \\ MSU, Moscow, Russia \\ alekseev@physics.msu.ru
}

\begin{abstract}
New methodology of cluster analysis of gene expression trends with averaging using a new moving average method was developed and applied to metabolically important genes in human PBMC cells. Gene expression trends were obtained from 5 independent datasets (total number of people was 2503, including 1551 males and 952 females), and an original algorithm was developed and implemented to harmonize them. An algorithm for detecting communities on an undirected graph was used, where genes were the nodes, and the edges represented strong direct and inverse correlation of age-related gene expression trends. Optimal values of the hyperparameters for the clustering procedure were found, and the averaging procedure that developed for the division of the genes into clusters. Nonmonotonic behavior with local minima and maxima was shown for the trends of expression of some genes, which may correspond to the transition processes between some stages of human aging.
\end{abstract}

Keywords - bioinformatics, human aging, RNA-seq data harmonization, PBMC, trend analysis, graph clustering

\section{Context and the goal}

\section{Introduction}

System biology research in the aging, especially human aging, is an urgent, but still underdeveloped area of modern bioscience. Currently, the primary omics data on biomarkers of aging and age-associated genes is insufficient for constructing a generalized theory of human aging, which would explain the facts on the pathophysiology of aging. So far, the aging can be defined as a set of underlying pathological interconnected processes at various levels of organization of human body [1].

A number of genes is known to be associated with human aging and the development of aging-related diseases [2]. However, the relationships between these processes and changes in the levels of gene expression has not been sufficiently studied. Even for genes and proteins with known association with lifespan, which signaling pathways are well studied (such as AMPK, the regulator of cell metabolism, or transcription factor $\mathrm{NF}-\kappa \mathrm{B}$, etc.), we do not see unambiguous relationships between gene expression change and the major pathological processes of aging, such as mitochondrial dysfunction, systemic insulin resistance, etc.). There are not many published papers on gene expression changes in various types of human cells or tissues during aging. This is due to the deficiency of open datasets on gene expression in human tissues or cells with large samples and age and sex indication.

Data analysis is also complicated by inter-individual, withinindividual and intersex variability, which leads to "fuzzy" distribution of the data on the graphs representing the changes of gene expression during aging. At the same time, average dynamics (trends) of expression of individual genes is of considerable interest, since it can allow us to associate the directions and patterns of such trend curves with the pathological processes of aging, changes in the tissue cellular composition, and the features of the extracellular environment (concentration of various metabolites, signaling molecules, etc.). Methods for clustering age-related trends for proteomic and metabolomic data have been published recently [3,4]. This report is one of the first analyses of age-related gene expression trends.

Research goals and tasks

The goal of this study is to identify the trends of expression of the key metabolic genes during aging, as well as the gene clusters based on the dynamics of expression of such genes. This requires solving several tasks:

- development of an approach to juxtapositioning data from various datasets (so-called "data harmonization");

- development of a method of averaging the merged data and obtaining gene expression trends for the set of metabolically important genes available in all datasets;

- development of a method for assessing the trends of correlation between the expression of each pair of genes;

- development and application of the methods of clusterization of gene expression trends (finding communities of correlated trends in the graph) to allow identification of the groups of gene with similar dynamics during aging;

- analyzing the clusters of the trends in expression of metabolically significant genes in human peripheral blood mononuclear cells (PBMCs).

\section{Methods}

337 genes from human PBMCs were analyzed (separately for men and women). The groups of genes of interest are related with the use or the production of NAD + and NADPH in cells, the Krebs cycle, glycolysis, pentose phosphate and glutathione metabolic pathways, as well as AMPK. The following method of identifying the gene expression trends and their clusterization was developed.

1. The used pipeline (a script on $\mathrm{R}$ language) allowed obtaining gene expression trends in PBMCs of healthy people using datasets from the GEO database [5], including GSE75511, GSE30483, GSE47353, GSE68759, and GSE65907, which contained information on sex and age of people. The dataset GSE65907 with a large sample and a wide age range was chosen as the reference. The data from men and women were merged for normalization. The normalization was performed for each gene from each dataset, except for the referential one (GSE65907), by comparing distribution density of the gene expression levels with that of the same genes in the reference dataset. The distribution turned out to be close to normal. The points for aligning the distribution densities were selected taking into account the differences in the distribution of people by age in each of considered and the reference datasets. Since age distribution was different in the sample, the samples of 28 randomly chosen individuals were selected for each dataset including the reference one for gene alignment.The samples of the selected individuals where only of these age ranges for which age distribution intersected on both sides of the distribution 
interval boundaries. A linear function was developed to calculate the normalized expression values for each gene ELnorm (gene) $=\mathrm{EL}$ (gene) $* \mathrm{k}+\mathrm{b}$, where the pairs of the parameters $(\mathrm{k}, \mathrm{b})$ were obtained by iterative minimization. Optim function of the standard set of the $\mathrm{R}$ language was used, and random pairs of numbers in a wide range of values were used as initial parameters values. The minimization target function was defined by the proximity between $10 \%$ and $90 \%$ quantiles in the reference dataset (in the age-limited samples, as described above) and $10 \%$ and $90 \%$ quantiles of normalized sample from a considered dataset. The sets of best pairs of parameters $(k, b)$ for each dataset and for each gene were obtained separately.

2. The averaging procedure was performed via a new, a specially developed moving average method with varying window width ( $\mathrm{R}$ code) to obtain a trend for each gene for the combined sample of the data points. The algorithm changes the moving averaging window so that the points from a certain age range fall into it, which was necessary because the distribution of different age groups varied from dataset to dataset. Moreover, the selection of the best pairs of parameters from each nonreference datasets and the reference dataset $(\mathrm{k}, \mathrm{b})$ was carried out randomly 20 times, and a set of the points normalized in different ways was formed. Thus, 20 sets of the pairs were obtained and processed by the moving average procedure. Each set was smoothed via cubic spline (standard R language smooth.spline function) and then, each ultimate gene expression trend was calculated as the average for the 20 smoothed trends.

3. The procedure of iterative calculation of the correlation coefficients in a moving window with a certain step was used to cluster the trends for each pair of genes. As a result, a set of correlation coefficients was obtained (ranging from -1 to 1 ), showing how the trend curves of the two genes were correlated in different age intervals. The distribution density of the correlation coefficients was calculated to identify the strongest correlations within the entire age range. Thus, strongly correlated pairs of the genes were identified, including direct and inverse correlations.

At this stage, the best number of clusters was estimated via hierarchical clustering and clustering of k-means using the Calinsky-Harabasz index [6]. The connection graph was constructed where the connections imply strong correlation of the gene expression trends. I searched for the communities in the graphs obtained from the data from men and women calculating the distribution of genes in the clusters based on the trends of their expression during aging as well as the modularity of graphs using several procedures of the iGraph library of the R language. Functions from d3Network R package were used for plotting the interactive graphs. Figure 1 (Supplementary materials link) shows the gene community graph which was produced based on the patterns of correlations between the genes, using community search methods.

\section{Results}

Age-related gene expression trends were calculated for the considered genes. Interestingly, the number of gene expression trends have distinct non-monotonic shape. Thus, the critical age points obtained for men were 34,43 , and 50 . The genes with minimum or maximum gene expression at the age of 43 may be associated with metabolic transition in men at this age point, associated with reduced ability to increase NAD+ levels in cells [7] (e.g., IDH3A, SQLE, SPARC, PPBP). Trends in expression of a group of genes display clear non-monotonous behavior near the age of 50 years (AASDHPPT, AGPAT2, DHCR7, MGST2, NDUFB1, NDUFA8, POR), that can be connected to significant increase in AMP concentration in the cells in 50 years [8]. However, the expression of the genes of AMPK subunits (PRKAA1, PRKAB1, PRKAB2, PRKAG1, PRKAG2) is not nonlinear at this age point. Strong correlation between the trends of a priori coexpressed genes during aging separately in men and women validates the new approach and the biological meaning of the results, as well as their similarity to the previously published data on gene expression trends for some of the genes.

\section{Discussion}

A large number of gene expression trends was obtained in this data analysis. Further detailed investigations and comparison of gene expression trends to the dynamics of the pathophysiological processes of aging, as well as to the metabolic and proteomic data. It is also necessary to validate the findings of this study with similar analysis of data from other tissues (muscles, liver, adipose tissue, etc.) to separate tissuespecific patterns of gene expression and the effects of aging. It would be also important to take into account the influence of cellular composition of PBMCs on age-related gene expression changes, since some gene expression patterns may be cellspecific. The development of such methods for gene expression analysis in various tissues in different phenotypic groups (different sexes, BMI levels, etc.) can open ways to create a unified omics databases on aging trends with different types of primary data, which would be a resource for developing system biology models of specific aging-related pathological processes and human aging as a whole.

\section{ACKNOWLEDGMENT}

The author is grateful to Daria Khaltourina for fruitful scientific discussions, as well as support in editing and text design.

\section{SUPPLEMENTARY MATERIALS}

The Figures and the tables are available online: https://yadi.sk/d/vdPAXhZ8c1C9Mg

\section{REFERENCES}

[1] Khaltourina, D., et al (2020) Aging Fits the Disease Criteria of the International Classification of Diseases. Mechanisms of Ageing and Developmen: 111230 .

[2] Tacutu, R. et al (2018) Human Ageing Genomic Resources: new and updated databases. Nucleic Acids Research. 46(D1): D1083-D1090.

[3] Lehallier, B. et al (2019) Undulating changes in human plasma proteome profiles across the lifespan. Nature Medicine. 25(12): 1843-1850.

[4] Bunning, B. J. et al (2020) Global metabolic profiling to model biological processes of aging in twins. Aging cell. 19(1): e13073.

[5] Gene Expression Omnibus - https:/www.ncbi.nlm.nih.gov/geo/

[6] Caliński, T., \& Harabasz, J. (1974) A dendrite method for cluster analysis. Communications in Statistics-theory and Methods. 3(1): 1-27.

[7] Clement, J. et al (2019) The plasma NAD+ metabolome is dysregulated in "normal" aging. Rejuvenation research, 22(2): 121-130.

[8] Ravera, S. et al (2019) Discrete changes in glucose metabolism define aging. Scientific reports. 9(1): 1-8. 